\title{
Relativistic self-focusing of intense laser beam in thermal collisionless quantum plasma with ramped density profile
}

\author{
S. Zare, ${ }^{1}$ E. Yazdani, ${ }^{2}$ S. Rezaee, ${ }^{1}$ A. Anvari, ${ }^{1}$ and R. Sadighi-Bonabi ${ }^{1, *}$ \\ ${ }^{1}$ Department of Physics, Sharif University of Technology, P.O. Box 11365-9567, Tehran, Iran \\ ${ }^{2}$ Department of Energy Engineering and Physics, Amirkabir University of Technology, \\ P.O. Box 15875-4413, Tehran, Iran
}

(Received 2 March 2014; published 20 April 2015)

\begin{abstract}
Propagation of a Gaussian x-ray laser beam has been analyzed in collisionless thermal quantum plasma with considering a ramped density profile. In this density profile due to the increase in the plasma density, an earlier and stronger self-focusing effect is noticed where the beam width oscillates with higher frequency and less amplitude. Moreover, the effect of the density profile slope and the initial plasma density on the laser propagation has been studied. It is found that, by increasing the initial density and the ramp slope, the laser beam focuses faster with less oscillation amplitude, smaller laser spot size and more oscillations. Furthermore, a comparison is made among the laser self-focusing in thermal quantum plasma, cold quantum plasma and classical plasma. It is realized that the laser self-focusing in the quantum plasma becomes stronger in comparison with the classical regime.
\end{abstract}

DOI: 10.1103/PhysRevSTAB.18.041301

PACS numbers: 52.38.Hb, 52.25.Os, 52.38.Kd, 41.75.Jv

\section{INTRODUCTION}

The construction of compact ultraintense and ultrashort laser pulses has become feasible by the chirped pulse amplification technique. Propagation of such laser pulses has been investigated in various plasma conditions $[1,2]$. Since the introduction of relativistic self-focusing effect by Hora in 1975 [3], improving the laser self-focusing in plasmas has become highly attractive due to its important role in the recent developments of the laser-plasma interaction, including monoenergetic electron generation, ion block acceleration and fast ignition of inertial confinement fusion (ICF) [4-6]. When an intense laser beam propagates in the plasma, due to the induced quivering motion of electrons resulting from the laser electric field, the plasma refractive index alters [3]. Subsequently, the plasma behaves initially similar to a positive lens that decreases the laser spot size [7-9]. In this process, producing relativistic electron oscillations and increasing the dielectric constant are desirable [3]. In order to achieve a better laser-plasma interaction, deeper penetration of high intensity beams in the plasma is favorable. In the classical regime, the laser self-focusing effect has been studied in homogenous and inhomogeneous plasmas by many researchers [10-13].

Recently, the high density and low temperature plasmas known as quantum plasmas are encountered in modern

\footnotetext{
*sadighi@sharif.ir

Published by the American Physical Society under the terms of the Creative Commons Attribution 3.0 License. Further distribution of this work must maintain attribution to the author(s) and the published article's title, journal citation, and DOI.
}

technology [14] and many environments, i.e., metallic nanoparticles [15], astrophysical systems [16], biophotonics [17], neutron stars [18], laser produced plasmas [19], fast ignition [20-25], quantum well and quantum diodes $[26,27]$. The classical and the quantum models for plasma are characterized by the thermal de Broglie wavelength, $\lambda_{B}=\hbar /\left(m_{e} k_{B} T\right)^{1 / 2}$, where $\hbar, m_{e}, k_{B}$ and $\mathrm{T}$ are the rationalized Planck's constant, the electron mass, the Boltzmann constant and the plasma temperature, respectively [15]. $\lambda_{B}$ roughly represents the spatial extension of the particle wave function by considering the quantum uncertainty. Therefore, the quantum effects are important when the de Broglie wavelength of electron is equal to or greater than the average interelectron distance, $n_{e}^{-1 / 3}$, i.e., $n_{e} \lambda_{B}^{3} \geq 1$, where $n_{e}$ represents the electron plasma density $[15,28]$. In the classical regime, the de Broglie wavelength is small enough to relinquish overlapping of the wave functions and quantum interferences; as a result, the particles could be considered as points. In other words, the quantum effects are important if the plasma temperature is equal to or less than the electron Fermi temperature. When the quantum effects are dominant, the relevant statistical distribution changes from Maxwell Boltzmann to Fermi Dirac. The Fermi temperature can be defined as follows [29]:

$$
\begin{gathered}
k_{B} T_{F}=E_{F}=\frac{\hbar^{2}}{2 m_{e}}\left(3 \pi^{2} n_{e}\right)^{2 / 3}, \\
\frac{T_{F}}{T}=\frac{1}{2}\left(3 \pi^{2}\right)^{2 / 3}\left(n_{e} \lambda_{\mathbf{B}}^{3}\right)^{2 / 3},
\end{gathered}
$$

where $T_{F}$ and $E_{F}$ represent the Fermi temperature and the Fermi energy, respectively. From Eq. (1), it is clear that the 
quantum effects in the plasma with lower temperature or higher density would be more remarkable.

Studying the quantum plasma requires new or modified models that are different from the current classical approach [15,28,30-32]. The first theoretical works have been reported by Klimontovich et al. [33], Bohm and Pines [34]. There are three outstanding methods for studying the quantum plasma, including Wigner-Poisson, SchrödingerPoisson and quantum hydrodynamic (QHD) models. The Wigner-Poisson and the Schrödinger-Poisson approaches describe the kinetic and the hydrodynamic behavior of plasma particles, respectively [30,35]. The QHD model similar to the classical fluid model is formulated by assuming velocity-space momentum of the Wigner equations. The QHD method is obtained by adding the Bohme potential as a quantum correction term in the plasma fluid model $[14,31,36]$. Anderson et al. presented a comparative study about the statistical behavior of the quantum plasma by the Wigner-Poisson approach [35]. Different phenomena have been studied in the various quantum plasmas; for instance, the nonlinear electron dynamics in thin metal films [37], the description of quantum diode in the dense Fermi magnetoplasma [38], breather mode in semiconductor quantum wells [39], parametric amplification characteristics in piezoelectric semiconductors [40], the plasmon excitation in nanowires [41], quantum ion-acoustic waves in unmagnetized quantum plasma [36] and single-walled carbon nanotubes [42].

The nonlinear phenomena are more noticeable in quantum plasmas in comparison with classical plasmas $[32,43]$. During the propagation of the laser beam in the quantum plasma, the laser spot size oscillates with higher frequency and less amplitude resulting in stronger laser self-focusing in comparison with the one in the classical regime [43-45]. In a quantum Fermi plasma, the feasible ranges of $n_{e}$ and $T_{F}$ are about $10^{18}-10^{23} \mathrm{~cm}^{-3}$ and $10^{7}-10^{9} \mathrm{~K}$, respectively, which are similar to the amounts for the compressed targets in the ICF scheme [46,47]. It is recognized that, by increasing the Fermi temperature, the quantum effects become more important [48].

The self-focusing of an intense laser beam in the classical plasma and in the cold quantum plasma has been studied by considering different plasma density profiles [49-52]. In the thermal homogenous quantum plasma, the laser selffocusing effect has been investigated by Patil et al. [48]. It has been realized by some experiments, in the interaction of the laser with the target, the plasma is created by the laser prepulse. By the expansion of the plasma away from the target-air interface, the plasma density increases from vacuum to the solid target that this density profile is called a ramped density [53]. Therefore, the ramped density profile is a better approximation of real plasma. There is much motivation to study the interaction of the relativistic laser intensities in thermal quantum plasma with the ramped density profile. More recently, laser self-focusing in the warm quantum plasma is reported by Habibi et al. [54], however, some parameters such as Fermi temperature and plasma density dimension are ignored.

In this paper, based on the defined plasma parameters, a ramped density profile is considered and the propagation of a relativistic Gaussian x-ray laser beam in the thermal collisionless quantum plasma is investigated. Using the Wentzel-Kramers-Brillouin (WKB) and paraxial approximations through the parabolic equation, a mathematical formulation for the beamwidth parameter in the thermal collisionless quantum plasma is obtained from the wave equation. Afterward, with considering the ramped density profile, the evolution of the beamwidth parameter along the propagation direction is presented. It is noticed that the laser self-focusing increases in the presence of the ramped plasma density more than the one in the uniform density. Moreover, the effect of the plasma parameters such as the ramp density slope and the initial plasma density on the laser self-focusing is investigated. It is found that in denser plasmas, the laser self-focusing occurs earlier with smaller minimum spot size and the laser beam width oscillates with the less amplitude and the higher frequency. Finally, the self-focusing effect in the thermal quantum plasma, the cold quantum plasma and the classical plasma is compared.

\section{THEORY}

The cylindrical coordinate system is used to investigate the propagation of a Gaussian laser beam along the $z$ axis. In this coordinate, the amplitude of the electric field, $E$, satisfies the scalar wave equation has the following form,

$$
\frac{\partial^{2} E}{\partial z^{2}}+\frac{1}{r} \frac{\partial}{\partial r}\left(r \frac{\partial E}{\partial r}\right)+\frac{\omega^{2}}{c^{2}} \varepsilon(r, z) E=0 .
$$

$\varepsilon(r, z), \mathrm{c}, \mathrm{r}$ and $\omega$ represent the dielectric constant of plasma, the speed of light in vacuum, the radial coordinate of the cylindrical system and the incident wave frequency, respectively. The solution of Eq. (2) is proposed by Akhmanov et al. [55] and Sodha et al. [56] as follows:

$$
E(r, t)=A(r, z) \exp \left(i \omega t-i \int_{0}^{z} k(z) d z\right) .
$$

$k=\sqrt{\varepsilon_{0 r}} \omega / c$ is the wave number where $\varepsilon_{0 r}$ is the linear part of the dielectric constant and $A(r, z)$ represents the complex amplitude. By assuming the WKB approximation for slowly converging or diverging beams and neglecting $\partial^{2} A / \partial z^{2}$, Eq. (4) is obtained by substituting Eq. (3) into Eq. (2),

$$
\begin{aligned}
2 i k(z) \frac{\partial A(r, z)}{\partial z}= & \frac{1}{r} \frac{\partial}{\partial r}\left(r \frac{\partial A(r, z)}{\partial r}\right) \\
& +\frac{\omega^{2}}{c^{2}}\left[\varepsilon(r, z)-\varepsilon_{0 r}\right] A(r, z) .
\end{aligned}
$$


$A(r, z)$ is composed of the real amplitude function, $A_{0}(r, z)$,

$$
A(r, z)=A_{0}(r, z) \exp [-i k(z) S(r, z)],
$$

and for the collisionless plasma, the eikonal function, $S(r, z)$, is defined as

$$
S(r, z)=S_{0}(z)+\frac{r^{2}}{2 f} \frac{d f}{d z},
$$

where $S_{0}$ and $f$ are the axial phase and the dimensionless beamwidth parameter, respectively. Substituting $A(r, z)$ from Eq. (5) into Eq. (4) by considering $S(r, z)$ as Eq. (6), and equating the real term of the resulted equation to zero, Eq. (7) is obtained:

$$
2 \frac{\partial S}{\partial z}+\left(\frac{\partial S}{\partial r}\right)^{2}=\frac{1}{k^{2} A_{0}}\left(\frac{\partial^{2} A_{0}}{\partial r^{2}}+\frac{1 \partial A_{0}}{r} \frac{\varepsilon r}{\partial r}\right)+\frac{\varepsilon-\varepsilon_{0 r}}{\varepsilon_{0 r}} .
$$

For the laser beams with the Gaussian intensity distribution, $A_{0}$ has the following form:

$$
A_{0}(r, z)=\frac{A_{00}}{f(z)} \exp \left(\frac{-r^{2}}{2 r_{0}^{2} f^{2}(z)}\right) .
$$

$A_{00}$ and $r_{0}$ are the initial electric field amplitude and the initial laser beam width, respectively. Considering the quantum mechanical effect, the Fermi gas pressure and the Bohm potential, the dielectric constant for unmagnetized and collisionless thermal quantum plasma takes this form [52,57]:

$$
\varepsilon=1-\frac{\omega_{p}^{2}}{\gamma\left(\omega^{2}-\gamma^{-1} \alpha k^{4}-k^{2} V_{F}^{2}\right)},
$$

where $\alpha=\hbar^{2} / 4 m_{e}{ }^{2}$ is the electron quantum diffraction due to the quantum correlation of density fluctuation. $\omega_{p}=$ $\sqrt{4 \pi e^{2} n_{e} / m_{e}}$ represents the electron plasma frequency where $e$ is the electron charge. $V_{F}=\sqrt{2 k_{B} T_{F} / m_{e}}$ and $\gamma=\sqrt{1+e^{2} E E^{*} /\left(m_{e}^{2} \omega^{2} c^{2}\right)}$ characterize the electron Fermi speed and the relativistic factor, respectively. Considering Eqs. (3), (5) and (8), the relativistic factor is equal to

$$
\gamma=\left[1+\frac{e^{2}}{m_{e}^{2} \omega^{2} c^{2}} \frac{A_{00}^{2}}{f^{2}} \exp \left(\frac{-r^{2}}{r_{0}^{2} f^{2}}\right)\right]^{1 / 2} .
$$

$\gamma$ can be expanded in a power series of $r^{2}$. Besides, in the paraxial approximation, the dielectric constant of nonabsorbing plasma can be presented as

$$
\varepsilon=\varepsilon_{0 r}+\varepsilon_{2 r} r^{2}
$$

where $\varepsilon_{0 r}$ and $\varepsilon_{2 r}$ are represented as functions of $z$,

$$
\begin{aligned}
& \varepsilon_{0 r}=1-\frac{\omega_{p}^{2}}{\omega^{2}}\left(1+\frac{e^{2}}{m_{e}^{2} \omega^{2} c^{2}} \frac{A_{00}^{2}}{f^{2}}\right)^{-1 / 2}\left[1-\frac{\alpha k^{4}}{\omega^{2}}\left(1+\frac{e^{2}}{m_{e}^{2} \omega^{2} c^{2}} \frac{A_{00}^{2}}{f^{2}}\right)^{-1 / 2}-\frac{k^{2} V_{F}^{2}}{\omega^{2}}\right]^{-1}, \\
& \varepsilon_{2 r}=-\frac{\omega_{p}^{2}}{\omega^{2}} \frac{e^{2}}{2 m_{e}^{2} \omega^{2} c^{2}} \frac{A_{00}^{2}}{r_{0}^{2} f^{4}}\left(1+\frac{e^{2}}{m_{e}^{2} \omega^{2} c^{2}} \frac{A_{00}^{2}}{f^{2}}\right)^{-3 / 2}\left[1-\frac{\alpha k^{4}}{\omega^{2}}\left(1+\frac{e^{2}}{m_{e}^{2} \omega^{2} c^{2}} \frac{A_{00}^{2}}{f^{2}}\right)^{-1 / 2}-\frac{k^{2} V_{F}^{2}}{\omega^{2}}\right] \\
& -1\left(1+\frac{\alpha k^{4}\left(1+\frac{e^{2}}{m_{e}^{2} \omega^{2} c^{2}} \frac{A_{00}^{2}}{f^{2}}\right)^{-1 / 2}}{\omega^{2}-\alpha k^{4}\left(1+\frac{e^{2}}{m_{e}^{2} \omega^{2} c^{2}} A_{00}^{2}\right)^{-1 / 2}-k^{2} V_{F}^{2}}\right) .
\end{aligned}
$$

By substituting Eqs. (6), (8) and (12) into Eq. (7) and equating the coefficient of $r^{2}$ to zero, the following equation is obtained:

$$
\frac{1}{f} \frac{d^{2} f}{d z^{2}}=\frac{1}{k^{2} f^{4} r_{0}^{4}}-\frac{p_{0} \omega_{p}^{2}}{2 \varepsilon_{0 r} r_{0}^{2} f^{4}} \frac{\left(1+\frac{p_{0}}{f^{2}}\right)^{-3 / 2}}{\omega^{2}-\alpha k^{4}\left(1+\frac{p_{0}}{f^{2}}\right)^{-1 / 2}-k^{2} V_{F}^{2}}\left(1+\frac{\alpha k^{4}\left(1+\frac{p_{0}}{f^{2}}\right)^{-1 / 2}}{\omega^{2}-\alpha k^{4}\left(1+\frac{p_{0}}{f^{2}}\right)^{-1 / 2}-k^{2} V_{F}^{2}}\right)
$$

$e^{2} A_{00}^{2} /\left(m_{e}^{2} \omega^{2} c^{2}\right)=p_{0}$ is the dimensionless quantity proportional to the laser beam power. It is better to express Eq. (13) in terms of the following dimensionless variables, $\xi$ and $\rho_{0}$ :

$$
\xi=z /\left(r_{0}^{2} k\right)=z /\left(2 R_{d}\right), \quad \rho_{0}=r_{0} \omega_{p} / c
$$


$\xi$ represents the dimensionless distance where $R_{d}$ is Rayleigh length. Assuming a Gaussian intensity distribution and an initial plane wave front, the boundary conditions are

$$
f=1, \quad d f / d \xi=0 \quad \text { at } \xi=0 .
$$

The dimensionless quantities of $\xi$ and $\rho_{0}$ from Eq. (14) are substituted into Eq. (13), and then Eq. (16) is the result:

$$
\begin{aligned}
\frac{d^{2} f}{d \xi^{2}}= & \frac{1}{f^{3}}-\frac{p_{0} \rho_{0}^{2} \omega^{2}}{2 f^{3}} \frac{\left(1+\frac{p_{0}}{f^{2}}\right)^{-3 / 2}}{\omega^{2}-\alpha k^{4}\left(1+\frac{p_{0}}{f^{2}}\right)^{-1 / 2}-k^{2} V_{F}^{2}} \\
& \times\left(1+\frac{\alpha k^{4}\left(1+\frac{p_{0}}{f^{2}}\right)^{-1 / 2}}{\omega^{2}-\alpha k^{4}\left(1+\frac{p_{0}}{f^{2}}\right)^{-1 / 2}-k^{2} V_{F}^{2}}\right) .
\end{aligned}
$$

The first term on the right-hand side of Eq. (16) explains the diffraction effect of the laser beam and the second nonlinear term shows the laser relativistic self-focusing. When $\left(d^{2} f / d \xi^{2}\right)_{\xi=0}$ is negative or positive values, the beam width will decrease (converge) or increase (diverge), respectively; and if $\left(d^{2} f / d \xi^{2}\right)_{\xi=0}$ is equal to zero, the beamwidth parameter would be constant through the propagation direction [58]. While from Eq. (16), for $\left(d^{2} f / d \xi^{2}\right)_{\xi=0}=0$, the critical condition could be obtained as

$$
\begin{aligned}
\rho_{0}^{2}= & \frac{2\left(1+p_{0}\right)^{3 / 2}}{p_{0}}\left(1-\frac{\alpha k^{4}\left(1+p_{0}\right)^{-1 / 2}}{\omega^{2}}-\frac{k^{2} V_{F}^{2}}{\omega^{2}}\right) \\
& \times\left(1+\frac{\alpha k^{4}\left(1+p_{0}\right)^{-1 / 2}}{2\left(\omega^{2}-\alpha k^{4}\left(1+p_{0}\right)^{-1 / 2}-k^{2} V_{F}^{2}\right)}\right)^{-1} .
\end{aligned}
$$

If the initial conditions of $p_{0}$ and $\rho_{0}$ satisfy Eq. (17), the induced self-focusing effect compensates the spatial diffraction; consequently, the laser beam propagates with constant beam width. If the initial value of $\rho_{0}$ is higher or lower than the amount obtained by Eq. (17) for a defined laser power, $\mathrm{p}_{0}$, the laser beam will be converged or diverged, respectively.

\section{RESULTS}

The quantum plasma condition in Eq. (9) is fulfilled when $\alpha k^{4} / \omega^{2} \gg 1$ i.e., $\omega$ should be large enough to satisfy this condition, accordingly, the laser wavelength is chosen in x-ray range [48]. To overcome the pulse diffraction and the extra consecutive amplitude oscillation of the spot size, the laser beam propagation is studied by considering a ramped plasma density. The inhomogeneous ramped density profile in one dimension is defined by $g(\xi)$ function while $B$ is a constant parameter [59],

$$
\begin{aligned}
g(\xi) & =\omega_{p}^{2} / \omega_{0 p}^{2}, \\
n_{e}(\xi) & =n_{0 e} g(\xi), \\
g(\xi) & =1+(B \xi)^{2} .
\end{aligned}
$$

$\omega_{0 p}=\sqrt{4 \pi e^{2} n_{0 e} / m_{e}}$ represents the initial electron plasma frequency where $n_{0 e}$ is the initial plasma density. From Eq. (18), it is deduced that with considering $B=2$, the electron density is 5 times higher than the initial electron density for $\xi=1$, i.e., $n_{e}=5 n_{0 e}$. Equation (16) is numerically solved by employing the fourth order Runge-Kutta method under the boundary conditions given by Eq. (15). To solve Eq. (16), the plasma and the laser parameters are considered as follows: $T_{F}=10^{9} \mathrm{~K}, n_{0 e}=4 \times 10^{19} \mathrm{~cm}^{-3}$, $B=2, \omega=1.778 \times 10^{20} \mathrm{~s}^{-1}, r_{0}=20 \mu \mathrm{m}$ and $\mathrm{p}_{0}=0.5$ $[46,48]$. For $\mathrm{p}_{0}=0.5$, the laser intensity equals $6.16 \times 10^{27} \mathrm{~W} \mathrm{~cm}^{-2}$, which presently is far beyond the accessible laser facilities that are required more technological advancement to be feasible in the future.

When the laser beam propagates in the collisionless plasma, the beam width steadily decreases because of the dominant relativistic self-focusing over the defocusing effect caused by the spatial diffraction. Moreover, due to the laser focusing into the plasma, the diffraction effect grows to become predominant and the beam width increases again. As a result, the laser spot size shows an oscillatory behavior.

Figure 1 denotes a comparison for the self-focusing effect in different plasma density profiles, the uniform density (solid curve) and the ramped density (dash curve). It is observed that initially the beamwidth parameter shows the similar oscillatory behavior for both density profiles along the propagation direction. With considering the ramped density profile, due to the plasma density enhancement, the laser self-focusing becomes stronger where the self-focusing length and the minimum laser spot size

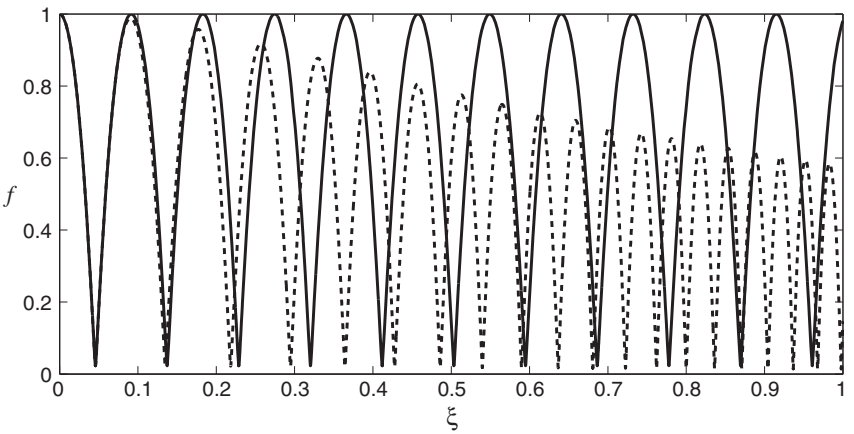

FIG. 1. Variation of the beamwidth parameter, $f$, with the dimensionless distance of propagation, $\xi=z /\left(2 R_{d}\right)$, for the ramped density profile with $B=2$ (dashed) and the uniform density $B=0$ (solid) at $T_{F}=10^{9} \mathrm{~K}, n_{0 e}=4 \times 10^{19} \mathrm{~cm}^{-3}, p_{0}=0.5, r_{0}=$ $20 \mu \mathrm{m}$ and $\omega=1.778 \times 10^{20} \mathrm{~s}^{-1}$. The laser propagation length at $\xi=1$ is equal to twice the Rayleigh length. 


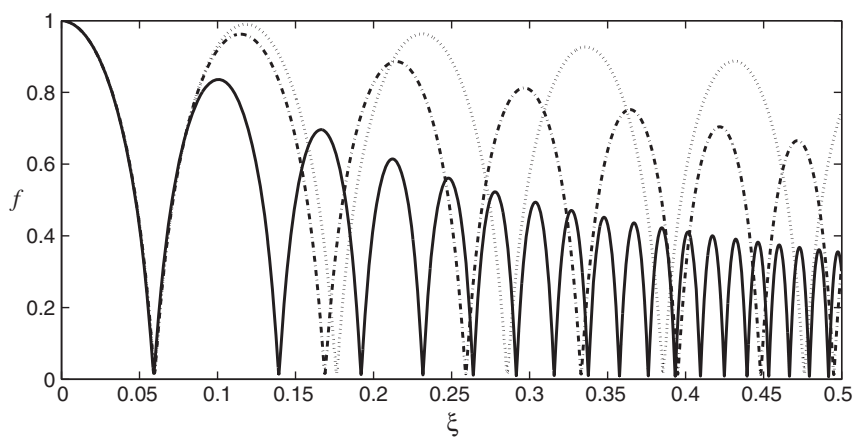

FIG. 2. Comparing the dimensionless beamwidth parameter for the propagation distance equal to one Rayleigh length, i.e., $\xi=0.5$ and in conditions of Fig. 1 for different density profile slopes, $B=1$ (dotted), $B=2$ (dash dotted) and $B=5$ (solid).

decrease. It is noticed at $\xi=1$, the laser spot size becomes less than half of the initial spot size, and as a result, the beam intensity in the plasma increases. At relativistic intensities, with increasing the plasma density, the electron beam with more relativistic electrons moves with the laser pulse. The higher electron current generates the higher quasistationery magnetic field; consequently, the pinching effect of the magnetic field becomes stronger, which further enhances the laser self-focusing. This prediction is reported by Pukhov et al. [60]. Also, the same results are presented for a ramped plasma density in the classical regime [51].

The dependence of the beamwidth parameter on the propagation distance for various ramped plasma density slopes, $B=1$ (dotted curve), $B=2$ (dash-dotted curve) and $B=5$ (solid curve) is shown in Fig. 2. For larger slopes, because of the plasma density enhancement, the beam width and the self-focusing length reduce along the propagation direction. Furthermore, the spot size decreases with a higher rate. In addition, it is shown that for the largest density slope $(B=5)$, the laser spot size becomes roughly smaller than $65 \%$ of its initial amount.

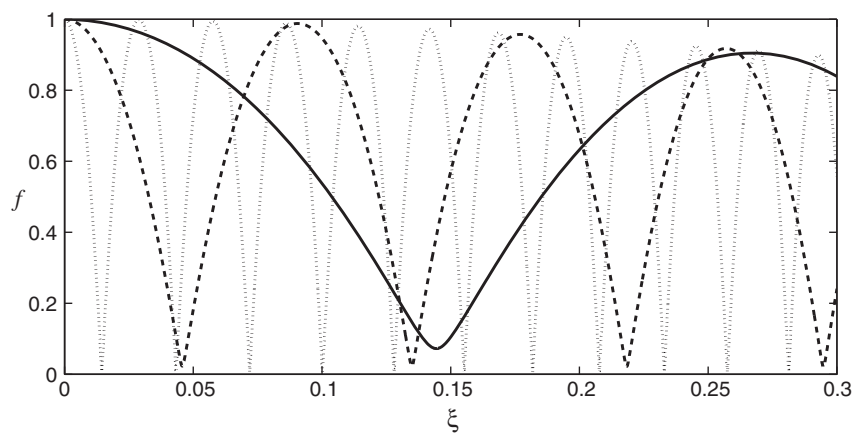

FIG. 3. Dependence of the beamwidth parameter on the dimensionless propagation distance and in conditions of Fig. 1 for different initial plasma densities of $n_{0 e}=4 \times 10^{18} \mathrm{~cm}^{-3}$ (solid), $n_{0 e}=4 \times 10^{19} \mathrm{~cm}^{-3}$ (dashed) and $n_{0 e}=4 \times 10^{20} \mathrm{~cm}^{-3}$ (dotted), with $B=2$.

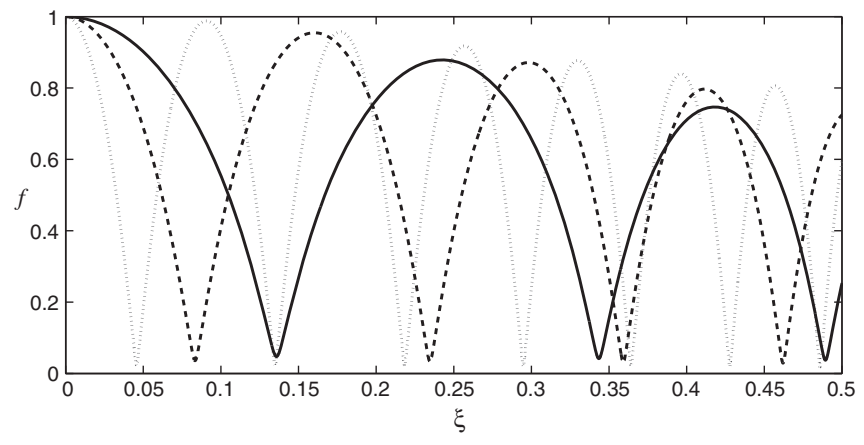

FIG. 4. Comparing the dimensionless beamwidth parameter with the dimensionless propagation distance for one Rayleigh length and in conditions of Fig. 1 for the thermal quantum plasma (dotted), the cold quantum plasma (dashed) and the classical plasma (solid), with $B=2$.

Figure 3 illustrates the beam width oscillation for various initial plasma densities of $n_{0 e}=4 \times 10^{18} \mathrm{~cm}^{-3}$ (solid curve), $n_{0 e}=4 \times 10^{19} \mathrm{~cm}^{-3}$ (dashed curve) and $n_{0 e}=4 \times 10^{20} \mathrm{~cm}^{-3}$ (dotted curve) with $B=2$. It is noticed that for denser plasmas, the oscillation amplitude and the minimum spot size decrease; therefore, the earlier and the faster laser self-focusing could be concluded; these results have been confirmed experimentally [61].

When the Fermi temperature is equated to zero, the thermal quantum plasma turns into the cold quantum plasma. In addition, if the Planck's constant is assumed to be zero, it is the same as ignoring quantum effects and switching from the quantum to the classical regimes. In Fig. 4, the laser self-focusing effect is compared for the thermal quantum plasma (dotted curve), the cold quantum plasma (dashed curve) and the classical case (solid curve). The quantum effects present the stronger pinching effect and consequently, the laser self-focusing in the quantum plasma becomes stronger. It is observed that in comparison with the classical plasma, for the thermal and cold quantum plasmas, the self-focusing length decreases about $66 \%$ and $40 \%$, respectively; also the minimum laser spot sizes are reduced by $54 \%$ and $23 \%$, respectively, in the first

TABLE I. The minimum dimensionless beamwidth parameter and the first self-focusing length obtained by considering the thermal and cold quantum models are compared with the ones given by the classical method in Fig. 4.

\begin{tabular}{lcccc}
\hline \hline & $\begin{array}{c}\text { Minimum } \\
\text { dimensionless } \\
\text { beam-width } \\
\text { Parameter }\left(f_{\text {min }}\right)\end{array}$ & $\begin{array}{c}\text { Improving } \\
\text { rate }\end{array}$ & $\begin{array}{c}\text { The first } \\
\text { self- } \\
\text { focusing } \\
\text { length }\end{array}$ & $\begin{array}{c}\text { Improving } \\
\text { rate }\end{array}$ \\
\hline $\begin{array}{l}\text { Classic } \\
\begin{array}{c}\text { Cold } \\
\text { quantum }\end{array}\end{array}$ & 0.0505 & & 0.1350 & \\
$\begin{array}{c}\text { Thermal } \\
\text { quantum }\end{array}$ & 0.0386 & $23 \%$ & 0.0837 & $46 \%$ \\
\hline \hline
\end{tabular}


cycle; refer to Table I, resulting from Fig. 4. Moreover, in the quantum model, the oscillation amplitude of the laser beam width is slightly larger than the classical case; this is due to the diffraction effect in the quantum plasma regime and disregarding some instability effects in the classical plasma model.

\section{CONCLUSION}

The propagation of the laser beam is studied based on the obtained differential equation for the beamwidth parameter. The ramped density profile is introduced for the laser selffocusing improvement in the relativistic thermal quantum plasma. For the ramped plasma density, the stronger laser self-focusing with the shorter focusing length is obtained in comparison with the uniform plasma density. In addition, it is noticed that, for the higher initial density and the larger ramp slope, earlier and faster self-focusing effect with smaller spot size could be achieved. Besides, the laser selffocusing in the thermal quantum plasma is compared with the one for the cold quantum and the classical plasma regimes. It is observed that in the quantum regime, the selffocusing length and the minimum beam width significantly decrease compared to the classical case.

The presented results could be useful in understanding the physics of high power laser driven fusion and clarifying the mechanism of the laser propagation in the quantum plasma that is important in the fast ignition of modern ICF experiments, where deuterium-tritium plasma is compressed by intense laser pulses and the quantum effects are dominant [62-64].

[1] E. Cormier-Michel, E. Esarey, C. G. R. Geddes, C. B. Schroeder, K. Paul, P. J. Mullowney, J. R. Cary, and W. P. Leemans, Phys. Rev. ST Accel. Beams 14, 031303 (2011).

[2] B. S. Rao, A. Moorti, P. A. Naik, and P. D. Gupta, Phys. Rev. ST Accel. Beams 16, 091301 (2013).

[3] H. Hora, J. Opt. Soc. Am. 65, 882 (1975).

[4] B. Hafizi, A. Tang, P. Sprangle, and R. F. Hubbard, Phys. Rev. E 62, 4120 (2000).

[5] P. Monot, T. Auduste, P. Gibbon, and F. Jakober, Phys. Rev. Lett. 74, 2953 (1995).

[6] I. Watts, M. Zepf, L. Clark, M. Tatarakis, K. Krushelnik, A. E. Dangor, R. Alott, J. Clarke, D. Neely, and P. N. Norreys, Phys. Rev. E 66, 036409 (2002).

[7] H. Hora, Laser Plasmas and Nuclear Energy (Plenum, New York, 1975), p. 20.

[8] H. Hora, Phys. Fluids 28, 3705 (1985).

[9] J. Faure, V. Malka, J.-R. Marquès, P.-G. David, F. Amiranoff, K. Ta Phuoc, and A. Rousse, Phys. Plasmas 9, 756 (2002).

[10] A. Upadhyay, V. K. Tripathi, A. K. Sharma, and H. C. Pant, J. Plasma Phys. 68, 75 (2002).

[11] M. Varshney, K. A. Qureshi, and D. Varshney, J. Plasma Phys. 72, 195 (2006).
[12] A. K. Sharma and A. Kourakis, Plasma Phys. Controlled Fusion 52, 065002 (2010).

[13] S. Kaur and A. K. Sharma, Laser Part. Beams 27, 193 (2009).

[14] P. K. Shukla, Nat. Phys. 5, 92 (2009).

[15] F. Hass, Quantum Plasmas: An Hydrodynamic Approach (Springer, New York, 2011).

[16] M. Opher, L. O. Silva, D. E. Dauger, V. K. Decyk, and J. M. Dawson, Phys. Plasmas 8, 2454 (2001).

[17] W. L. Barnes, A. Dereux, and T. W. Ebbesen, Nature (London) 424, 824 (2003).

[18] G. Chabreier, F. Douchin, and A. Y. Potekhin, J. Phys. Condens. Matter 14, 9133 (2002).

[19] K. H. Becker, K. H. Schoenbach, and J. G. Eden, J. Phys. D 39, R55 (2006).

[20] S. X. Hu and C. H. Keitel, Phys. Rev. Lett. 83, 4709 (1999).

[21] A. V. Andreev, J. Exp. Theor. Phys. Lett. 72, 238 (2000).

[22] P. K. Shukla and L. Stenflo, Phys. Plasmas 13, 044505 (2006).

[23] H. Azechi, Plasma Phys. Controlled Fusion 48, B267 (2006).

[24] M. Marklund and P. K. Shukla, Rev. Mod. Phys. 78, 591 (2006).

[25] S. H. Glenzer and R. Redmer, Rev. Mod. Phys. 81, 1625 (2009).

[26] L. K. Ang, W. S. Koh, Y. Y. Lau, and T. J. T. Kwan, Phys. D Plasmas 13, 056701 (2006).

[27] L. K. Ang and P. Zhang, Phys. Rev. Lett. 98, 164802 (2007).

[28] P. K. Shukla and B. Eliasson, Plasma Phys. Controlled Fusion 52, 124040 (2010).

[29] L. D. Landau and E. M. Lifshitz, Statistical Physics (Butterworth-Heinemann, Oxford, 1980), Pt. 1.

[30] P. K. Shukla and B. Eliasson, Phys. Rev. Lett. 96, 245001 (2006).

[31] G. Manfredi and F. Haas, Phys. Rev. B 64, 075316 (2001).

[32] P. K. Shukla and B. Eliasson, Phys. Usp. 53, 51 (2010).

[33] Y. L. Klimontovich and V. P. Silin, Doklady. Akad. Nauk. S. S. S. R. 82, 361 (1952).

[34] D. Bohm, Phys. Rev. 92, 609 (1953).

[35] D. Anderson, B. Hall, M. Lisak, and M. Marklund, Phys. Rev. E 65, 046417 (2002).

[36] F. Haas, L. G. Garcia, J. Goedert, and G. Manfredi, Phys. Plasmas 10, 3858 (2003).

[37] N. Crouseilles, P. A. Hervieux, and G. Manfredi, Phys. Rev. B 78, 155412 (2008).

[38] P. K. Shukla and B. Eliasson, Phys. Rev. Lett. 100, 036801 (2008).

[39] F. Haas, G. Manfredi, P. K. Shukla, and P. A. Hervieux, Phys. Rev. B 80, 073301 (2009).

[40] S. Ghosh, S. Dubey, and R. Vanshpal, Phys. Lett. A 375, 43 (2010).

[41] S. Ali, H. Tercas, and J. T. Mendonca, Phys. Rev. B 83, 153401 (2011).

[42] L. Wei and Y. N. Wang, Phys. Rev. B 75, 19340 (2007).

[43] P. K. Shukla, S. Ali, L. Stenflo, and M. Marklund, Phys. Plasmas 13, 112111 (2006). 
[44] M. Marklund and G. Brodin, Phys. Rev. Lett. 98, 025001 (2007).

[45] S. V. Bulanov, T. Zh. Esirkepov, D. Habs, F. Pegoraro, and T. Tajima, Eur. Phys. J. D 55, 483 (2009).

[46] V. M. Malkin, N. J. Fisch, and J. S. Wurtele, Phys. Rev. E 75, 026404 (2007).

[47] T. Farid, A. M. Mirza, P. K. Shukla, and A. Qamar, Phys. Plasmas 8, 846 (2001).

[48] S. D. Patil and M. V. Takale, Phys. Plasmas 20, 072703 (2013).

[49] S. D. Patil, M. V. Takale, S. T. Navare, M. B. Dongare, and V. J. Fulari, Optik (Stuttgart) 124, 180 (2013).

[50] M. Habibi and F. Ghamari, Phys. Plasmas 19, 103110 (2012).

[51] R. Sadighi-Bonabi, M. Habibi, and E. Yazdani, Phys. Plasmas 16, 083105 (2009).

[52] S. C. Na and Y. D. Jung, Phys. Plasmas 16, 074504 (2009).

[53] M. Burza, A. Gonoskov, K. Svensson, F. Wojda, A. Persson, M. Hansson, G. Genoud, M. Marklund, C. G. Wahlström, and O. Lundh, Phys. Rev. ST Accel. Beams 16, 011301 (2013).
[54] M. Habibi and F. Ghamari, Phys. Plasmas 21, 052705 (2014).

[55] S. A. Akhmanov, A. P. Sukhorukov, and R. V. Khokhlov, Sov. Phys. Usp. 10, 609 (1968).

[56] M. S. Sodha, A. K. Ghatak, and V. K. Tripathi, Selffocusing of Laser Beams in Dielectrics, Plasma and Semiconductors (Tata McGraw-Hill, New Delhi, 1974).

[57] S. Ali and P. K. Shukla, Phys. Plasmas 13, 102112 (2006).

[58] Y. Wang and Z. Zhou, Phys. Plasmas 18, 043101 (2011).

[59] S. Sen, B. Rathore1, M. Varshney, and D. Varshney, J. Phys. Conf. Ser. 208, 012088 (2010).

[60] A. Pukhov and J. Meyer-ter-vehn, Phys. Rev. Lett. 76, 3975 (1996).

[61] S. Y. Chen, G. S. Sarkisov, A. Maksimchuk, R. Wagner, and D. Umstadter, Phys. Rev. Lett. 80, 2610 (1998).

[62] S. Son and N. J. Fisch, Phys. Rev. Lett. 95, 225002 (2005).

[63] M. Tabak et al., Phys. Plasmas 12, 057305 (2005).

[64] Yu. Tyshetskiy, S. V. Vladimirov, and R. Kompaneets, Phys. Plasmas 18, 112104 (2011). 